\title{
A novel, low-cost and practical illumination approach for bimanual vitrectomy
}

\author{
(1) Veysel Aykut, (1) Fehim Esen, (1) Halit Oguz \\ Department of Ophthalmology, Istanbul Medeniyet University Faculty of Medicine, Istanbul, Turkey
}

\begin{abstract}
OBJECTIVE: The present study aims to describe a novel, low-cost, transconjunctival sutureless bimanual vitrectomy illumination approach and evaluate its surgical outcome.

METHODS: Thirty-six eyes of 36 patients who underwent pars plana vitrectomy with the above-mentioned technique were included in this study. Four trocars were placed in superotemporal, inferotemporal, inferonasal and superonasal quadrants. A piece $(23 \mathrm{~mm})$ was cut from $30 \mathrm{G}$ intravenous cannula and a $30 \mathrm{~mm}$ endoillumination probe was placed inside this sleeve. This design limited the entry of the light probe into the vitreous cavity to $7 \mathrm{~mm}$ and provided a safe illumination by the assistant without the risk of damaging the retinal tissue.

RESULTS: Thirty-six eyes of 36 patients were included ( 24 male, 12 female, mean age: $58.4 \pm 14.3$ years) in this study. Thirty patients had rhegmatogenous retinal detachment (six of these patients with coexisting choroidal detachment), four patients had diabetic tractional retinal detachment, one patient had a nucleus drop and one patient had an intraocular foreign body. The mean follow-up time after pars plana vitrectomy (PPV) was 5.05 \pm 4.4 months. LogMAR best-corrected visual acuity improved significantly after PPV $(p<0.001)$. Postoperative complications included recurrent detachment in two eyes, hypotony in one eye and endophthalmitis in one eye. The transient rise in intraocular pressure was observed in 19 patients, but there was no significant difference between the preoperative and postoperative mean IOP in the long term follow-up.

CONCLUSION: This bimanual vitrectomy system provided favorable outcomes without increasing the cost of standard PPV. The advantage of this system over the chandelier illumination include the reduction in glare, the possibility to change the direction of the light during the surgery to better illuminate the surgical site and the lower cost. This approach can be used in any vitrectomy system (20G, 23G, 25G, and 27G) by changing the size of the IV cannula.
\end{abstract}

Keywords: Bimanual vitrectomy; endoillumination; vitrectomy.

Cite this article as: Aykut V, Esen F, Oguz H. A novel, low-cost and practical illumination approach for bimanual vitrectomy. North Clin Istanb 2020;7(3):275-279.

$\mathrm{P}$ roper illumination of the surgical site is a must in vitreoretinal surgery. Therefore, the development of the first endoillumination probe revolutionized vitreoretinal surgery around forty years ago [1]. Since then, many developments have been achieved in both vitreoretinal surgery illumination systems and instruments.
Subsequently, the surgical outcome has become better and better over time [2].

Patients with proliferative vitreoretinopathy (PVR) are especially challenging cases and it is very hard to manage these cases with standard approaches. The development of chandelier light systems allowed biman-

This work was previously presented at the $52^{\text {nd }}$ National Ophthalmology Congress organized by Turkish Ophthalmological Society on November 2018 in Antalya.

Received: September 02, 2019 Accepted: January 21, 2020 Online: February 11, 2020

Correspondence: Dr. Veysel AYKUT. Istanbul Medeniyet Universitesi, Goztepe Egitim ve Arastirma Hastanesi, Goz Hastaliklari Klinigi, Kadikoy, Istanbul, Turkey.

Tel: +90 5054534570 e-mail: v-aykut@hotmail.com

(c) Copyright 2020 by Istanbul Provincial Directorate of Health - Available online at www.northclinist.com 
ual surgery in these cases, which made peeling of sticky tractional membranes relatively easier and contributed to the improvement of surgical outcomes in these cases [3]. Chandelier illumination systems can also be useful in other surgeries, such as scleral buckle surgery, cataract surgery of patients with corneal haze or intense vitreous hemorrhage [4-6]. There are three important limitations regarding the use of chandelier illumination systems. First, the system is relatively expensive. Thus, it cannot be used easily in low resource environments, such as in developing countries $[2,3,7,8]$. The second disadvantage of this system is the fixed location, which limits its success in the visualization of the retina. In the chandelier illumination system, the light source is not sheathed and therefore, glare is also an important problem impairing the visualization of the retina.

We developed a new illumination approach for bimanual vitrectomy to overcome the above-mentioned problems. In our four-port bimanual vitrectomy illumination system, we first insert a trocar in all four quadrants. We produce sleeves from the intravenous cannula and insert sheathed illumination probes into it. The sleeves limit the movement of the illumination probe into the vitreous cavity to $2 \mathrm{~mm}$ and the assistant can illuminate the surgical site safely by directing this light source trough any of the four vitrectomy ports. The present study aims to define this novel illumination approach for bimanual vitrectomy and share the results of surgeries performed with this technique.

\section{MATERIALS AND METHODS}

The records of thirty-six eyes of 36 patients, who had bimanual vitrectomy with the above-mentioned lowcost, four-port vitreoretinal surgery illumination system between the years of 2016 and 2018, were retrospectively studied. This study was approved by the local institutional ethics committee (document no: 2019/0027, date: 06.02.2019). All of the patients had a detailed ophthalmologic examination, including evaluation of best-corrected visual acuity (BCVA), slit-lamp examination, intraocular pressure (IOP) measurement with Goldmann applanation tonometry, and dilated fundus examination with indirect ophthalmoscopy.

The illumination system consisted of a trocar, an intravenous cannula and an endoillumination probe with beveled sheath design (Fig. 1). We shortened the length of the intravenous cannula to $23 \mathrm{~mm}$. The length of the endoillumination probe was $30 \mathrm{~mm}$ (Alcon, Irvine, CA,
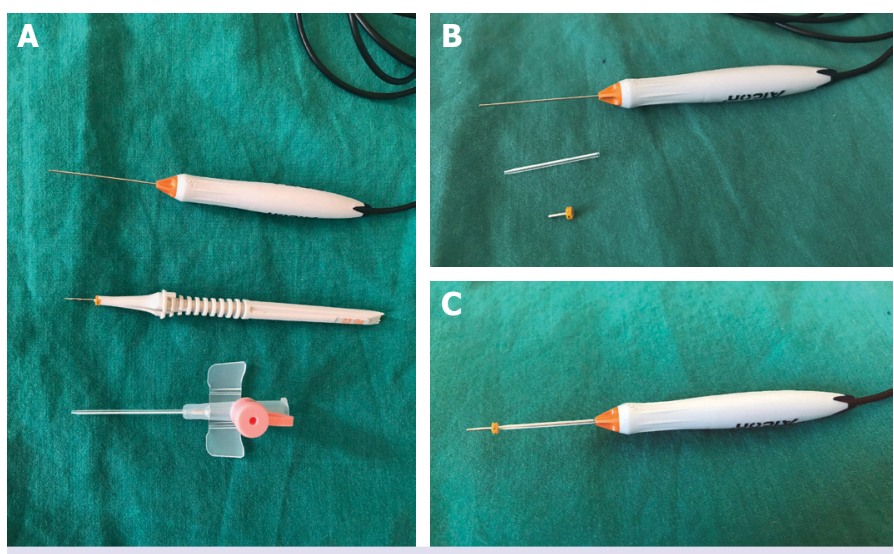

FIGURE 1. The preparation of the low-cost, bimanual pars plana vitrectomy illumination system. (A) The instruments needed for the system are displayed: A sheathed endoillumination probe, a vitrectomy trocar, an intravenous cannula at the same lumen size with the vitrectomy system (e.g., 23G, 25G, 27G) (B) The tip of the intravenous cannula is shortened to a size $7 \mathrm{~mm}$ shorter than the illumination probe. Here, the length of the illumination probe was 30 $\mathrm{mm}$, the length of the sheath was $23 \mathrm{~mm}$ and the length of the trocar was around $5 \mathrm{~mm}$ with its tip. (C) The illumination system is seen from the side. Around $2 \mathrm{~mm}$ of the illumination probe is exposed inside the eye (outside the trocar), and the risk of ocular trauma is minimal, even when it is manipulated by a relatively inexperienced assistant during surgery.

USA). Therefore, $7 \mathrm{~mm}$ of the endoillumination probe was exposed and $5 \mathrm{~mm}$ of it remained within the trocar. As a result, only $2 \mathrm{~mm}$ of the endoillumination probe could be inserted into the vitreous cavity to provide illumination for bimanual vitrectomy. Because the intraocular segment of the illumination probe was too short to damage the retinal tissue, the probe could be directed safely by the assistant during the surgery. This endoillumination system provided adequate illumination for pars plana vitrectomy and the beveled sheath design prevented glare very effectively when the light is directed posteriorly.

During the surgery, four $23 \mathrm{G}$ trocars were inserted transconjunctivally at four quadrants. Any of the four trocars could be used for infusion or illumination during the surgery. This flexibility in changing the position of the instruments was also an advantage for the surgeon in challenging cases. The illumination probe was directed to the surgical site by the assistant while the surgeon performed bimanual vitrectomy (Fig. 2). It was also possible for the surgeon to perform scleral indentation and vitrectomy or endolaser treatment at the same time, which was 

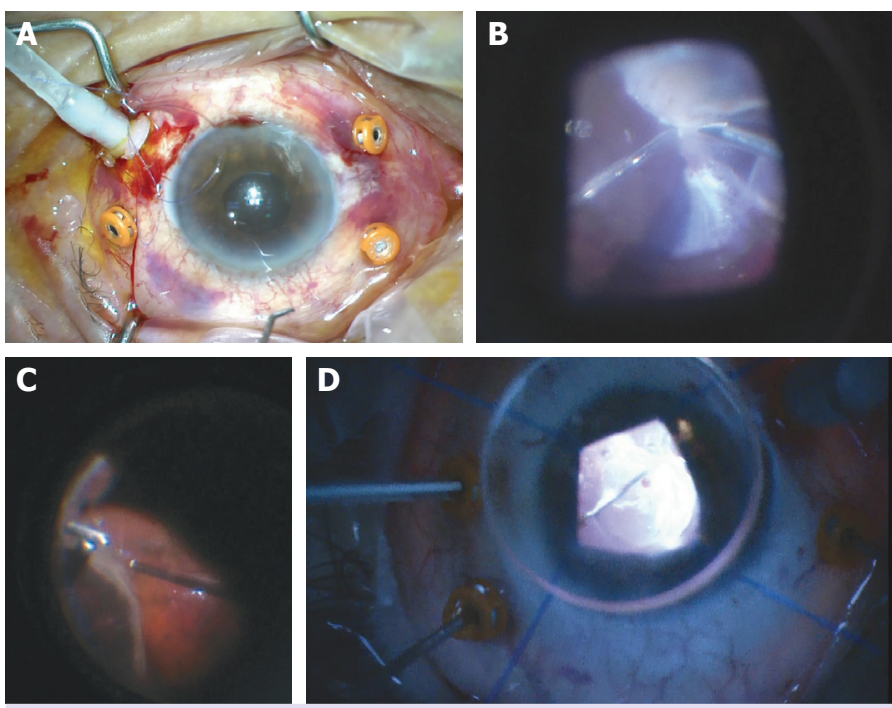

FIGURE2. Clinical images demonstrating the use of the lowcost, bimanual pars plana vitrectomy illumination system. (A) Four trocars are inserted through the scleral tissue in a manner similar to a regular pars plana vitrectomy trocar placement. (B) The assistant could safely direct the illumination probe to the surgical site, when the surgeon performed bimanual membrane peeling. (C) The surgeon could perform indentation and peripheral vitreous shaving with both hands, while the assistant illuminated the surgical site. (D) The clinical image of the surgery from the operating microscope. The sheathed illumination probe can be seen in the inferotemporal sclerotomy site, and the instruments for bimanual vitrectomy are inserted from the superotemporal and superonasal sclerotomies. The position of the tools and illumination probes can be freely switched during the surgery to provide easier access to the surgical site.

a very good advantage during cases that needed extensive vitreous base shaving. In nucleus drop cases, the nucleus was fragmented with any instrument other than the endoillumination probe, which also prevented the loss of illumination during the nucleus segmentation phase of the surgery. In cases with intraocular foreign bodies, the foreign body could be held bimanually by the surgeon, and the removal was relatively easier. In patients with choroidal detachment, one of the four trocars could be initially used for the drainage of the suprachoroidal fluid while remaining trocars were sufficient for a regular PPV.

\section{Statistical Analysis}

The data were analyzed using IBM SPSS software v21.0 for Mac (IBM Corp., Chicago, IL, USA). The distribution of the data was determined with Kolmogarov -
TABLE 1. Baseline clinical characteristics of the patients

\begin{tabular}{lccc} 
& $\mathrm{n}$ & $\%$ & Mean \pm SD \\
\hline Mean age (years \pm SD) & & & $58.4 \pm 14.3$ \\
Mean follow-up time (months \pm SD) & & & $5.05 \pm 4.4$ \\
Gender (m:f) & $24: 12$ & & \\
Diagnosis & & & \\
$\quad$ Rhegmatogenous retinal detachment & 30 & 83.33 \\
$\quad$ Diabetic tractional retinal detachment & 4 & 11.11 & \\
$\quad$ Nucleus drop & 1 & 2.77 \\
$\quad$ Intraocular foreign body & 1 & 2.77
\end{tabular}

SD: Standard deviation; m: Male; f: Female.

Smirnov test. Data were presented as mean \pm SD. Preoperative and postoperative findings were compared with repeated samples t-test. Snellen visual acuities were converted to LogMAR before statistical comparisons.

\section{RESULTS}

Thirty-six eyes of 36 patients ( 24 male, 12 female, mean age: $58.4 \pm 14.3$ years) who underwent PPV studied. The most common pathology was rhegmatogenous retinal detachment $(n=30)$ and six of them had coexisting choroidal detachment. Mean follow-up time was $5.05 \pm 4.4$ months. The baseline characteristics of the patients are described in detail in Table 1. Snellen best-corrected visual acuity (BCVA) improved significantly after $\operatorname{PPV}(0.34 \pm 0.49$ vs. $0.065 \pm 0.1, \mathrm{p}<0.001)$.

Complications included recurrent retinal detachment due to proliferative vitreoretinopathy (PVR) in two eyes, hypotony in one eye and endophthalmitis in one eye. The patients who had recurrent retinal detachment developed new retinal tears in the peripheral retina due to PVR. These eyes were operated again, subretinal fluid was drained through the retinal tear and laser retinopexy was performed around the retinal break. A patient with massive suprachoroidal hemorrhage secondary to trauma developed ocular hypotony during the postoperative follow-up. There was only one case of postoperative endophthalmitis, which could be treated successfully with a single intravitreal antibiotic injection $(\mathrm{mg} / 0.1$ $\mathrm{ml}$ vancomycin and $\mathrm{mg} / 1 \mathrm{ml}$ ceftazidime). There was a transient rise in intraocular pressure in 19 patients during the postoperative 24 hours. However, there was no significant difference between the preoperative and 
postoperative IOP values during long term follow-up. $(13.5 \pm 2.35$ vs. $13.9 \pm 3.7 \mathrm{mmHg}, \mathrm{p}=0.53)$. None of the above-mentioned complications were related to the preferred illumination system.

\section{DISCUSSION}

The chandelier illumination system is an essential tool for current bimanual vitreoretinal surgery. This system provides panoramic illumination to facilitate better visualization of the surgical site. However, this illumination pattern also causes glare, especially after the fluidair exchange, and laser photocoagulation is especially harder with this system. Our system uses regular beveled illumination probes. Different from the chandelier system, in our illumination system, glare is very rare as the beveled design of the probe prevents light scattering. The reduction in glare facilitates many stages of surgery, including laser photocoagulation and vitreous base shaving and reduces surgical time, according to our observations. Beveled probes illuminate retinal tissue locally and also enhance depth perception. Enhanced depth perception facilitates dissection of epiretinal membranes and the risk of retinal damage might be reduced during membrane dissection.

Many surgeons use endoillumination probe for chopping the dropped nucleus fragment, but visualization of the posterior segment is impaired during this maneuver and it has the potential to cause iatrogenic retinal damage. In our bimanual surgery system, chopping can be performed with a second instrument that is inserted through the fourth trocar. In patients with intraocular foreign bodies, the hardest and most challenging step is to hold the foreign body without causing new damage in the retinal tissue. The enhanced depth perception in our system also facilitated the safer removal of intraocular foreign bodies. The presence of a fourth trocar is also useful in patients with choroidal detachment with suprachoroidal hemorrhage/fluid accumulation. This suprachoroidal fluid can be drained through the fourth trocar, while the instrument inserted through the other trocars can be used to continue regular vitrectomy.

In chandelier illumination systems, the probe needs to be fixated in the scleral tissue trough one or two incisions according to the design of the system and these sclerotomies can only be used for illumination [7,9]. However, our system needs only one additional sclerotomy, and this port provides more flexibility during the surgery by allowing the insertion of different surgical instruments.
Our system can be used in any size of vitrectomy system (20G, 23G, 25G and 27G) and does not require any additional instrument for fixation.

The commercially available chandelier systems are generally inserted at the beginning of the surgery, although they are only needed for the bimanual part of the surgery [10]. Therefore, the surgeon suffers from the loss of comfort due to the presence of one or two extra cables during most parts of the surgery, when they are actually not needed. These cables restrict access to the surgical area, can hook the surgical instruments and can even be accidentally removed or displaced by an unwanted maneuver of the surgeon [11]. When these light fibers are removed accidentally, it is very hard to find the sclerotomy under the conjunctiva, especially in the presence of chemosis or subconjunctival hemorrhage [3]. Many different chandelier systems are developed to overcome the above-mentioned problems, but they are expensive and their fixation systems are inconvenient and timeconsuming $[11,12]$.

Chandelier light systems are inserted with a twostep technique. After the formation of the sclerotomy with the specific needle, there is always a risk of subconjunctival hemorrhage and loss of the visualization of the sclerotomy site. In this case, the surgeon needs to dissect the conjunctiva and search for the sclerotomy site, which prolongs surgical time and increases surgical trauma [12]. Our system can be inserted with a singlestep approach, as in a regular trocar insertion, without the above-mentioned problems. The tip of the chandelier light fibers can be coated with hemorrhage during surgery and the cleaning of these tips are relatively hard and time-consuming as they are fixated within the scleral tissue [8]. In our system, the endoillumination probe can be freely removed and cleaned as in a regular vitrectomy case.

Regular chandelier probes were reported to cause scleral thermal injury in a case because the heat of the light probe increases excessively during the fluid-air exchange. Some surgeons suggest the use of the regular chandelier light probes with a cannula to serve as a sheath and avoid thermal injury [10]. Chandelier light systems are especially needed in cases that need bimanual vitrectomy and extensive peripheral laser retinopexy under air [13]. Therefore, the risk of scleral thermal injury is increased. In the current study, the use of the endoillumination probe is quite similar to regular vitrectomy cases and does not increase the risk of thermal damage. 
The illumination probes need to be kept at a certain distance from the retinal tissue, and the exposure time should be short to prevent phototoxic damage [8, 13]. Therefore, continuous illumination of the retinal tissue is an unwanted illumination approach. Contrary to chandelier systems, the light probe illuminates only the surgical site instead of the entire retina. The direction of the probe can be safely changed by the assistant due to limited insertion length of the probe, and the probe cannot be too close to the retinal tissue, which prevents the risk of both mechanical and phototoxic damage. Further studies are needed to better document the advantages and disadvantages of this illumination approach.

Ethics Committee Approval: Medeniyet University Goztepe Training and Research Hospital Clinical Research Ethics Committee granted approval for this study (date: 06.02.2019, number: 2019/0027).

Conflict of Interest: No conflict of interest was declared by the authors.

Financial Disclosure: The authors declared that this study has received no financial support.

Authorship Contributions: Concept - VA, FE, HO; Design - VA, $\mathrm{FE}$, HO; Supervision - HO; Materials - VA; Data collection and/or processing - VA, FE; Analysis and/or interpretation - VA, FE, HO; Literature review - VA, FE, HO; Writing - VA, FE; Critical review - HO.

\section{REFERENCES}

1. Peyman GA. Improved vitrectomy illumination system. Am J Ophthalmol 1976;81:99-100. [CrossRef]
2. Mohamed S, Claes C, Tsang CW. Review of Small Gauge Vitrectomy: Progress and Innovations. J Ophthalmol 2017;2017:6285869.

3. Eckardt C, Eckert T, Eckardt U. 27-gauge Twinlight chandelier illumination system for bimanual transconjunctival vitrectomy. Retina 2008;28:518-9. [CrossRef]

4. Jang SY, Choi KS, Lee SJ. Chandelier retroillumination-assisted cataract extraction in eyes with vitreous hemorrhage. Arch Ophthalmol 2010;128:911-4. [CrossRef]

5. Aras C, Ucar D, Koytak A, Yetik H. Scleral buckling with a non-contact wide-angle viewing system. Ophthalmologica 2012;227:107-10.

6. Yamamoto N, Ozaki N, Murakami K. Trypan-blue- and endoillumination-assisted phacoemulsification in eyes with vitreous hemorrhage during combined cataract and vitreous surgery. Ophthalmologica 2005;219:338-44. [CrossRef]

7. Eckardt C. Twin lights: a new chandelier illumination for bimanual surgery. Retina 2003;23:893-4. [CrossRef]

8. Koch FH, Pawlowski D, Spitznas M. A multiport illumination system for panoramic bi-manual vitreous surgery. Graefes Arch Clin Exp Ophthalmol 1991;229:425-9. [CrossRef]

9. Kellner L, Wimpissinger B, Stolba U, Brannath W, Binder S. 25-gauge vs 20-gauge system for pars plana vitrectomy: a prospective randomised clinical trial. Br J Ophthalmol 2007;91:945-8. [CrossRef]

10. Shimada H, Nakashizuka H, Hattori T, Mori R, Mizutani Y. Thermal injury caused by chandelier fiber probe. Am J Ophthalmol 2007;143:167-9. [CrossRef]

11. Sakaguchi H, Oshima Y, Nishida K, Awh CC. A 29/30-gauge dualchandelier illumination system for panoramic viewing during microincision vitrectomy surgery. Retina 2011;31:1231-3. [CrossRef]

12. Uemoto R, Nakasato S, Mizuki N. New technique for inserting 27 -gauge twinlight chandelier illumination fibers into the eye using intravenous cannula. Retina 2009;29:1040-2. [CrossRef]

13. van den Biesen PR, Berenschot T, Verdaasdonk RM, van Weelden $H$, van Norren D. Endoillumination during vitrectomy and phototoxicity thresholds. Br J Ophthalmol 2000;84:1372-5. [CrossRef] 